\title{
Susceptibilidad ambiental a la desertificación en la microcuenca del río Azumiatla, Puebla, México
}

\section{Environmental susceptibility to desertification in the Azumiatla River Micro-watershed, Puebla, Mexico}

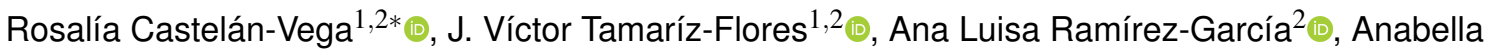 \\ Handal-Silva ${ }^{2,3}$ (D), Wendy Argelia García-Suastegui ${ }^{2,3}$ (D) \\ ${ }^{1}$ Departamento de Investigación en Ciencias Agrícolas, Benemérita Universidad Autónoma de Puebla. 14 Sur 6301, Colonia San Manuel, \\ CP. 72470. Puebla, Puebla, México. \\ ${ }^{2}$ Posgrado en Ciencias Ambientales, Benemérita Universidad Autónoma de Puebla. 14 Sur 6301, Colonia San Manuel, CP. 72470. \\ Puebla, Puebla, México. \\ ${ }^{3}$ Laboratorio de Toxicología Ambiental, Departamento de Biología y Toxicología de la Reproducción, Instituto de Ciencias, Benemérita \\ Universidad Autónoma de Puebla. 14 Sur 6301, Colonia San Manuel, CP. 72470. Puebla, Puebla, México. \\ *Autor de correspondencia: rosalia.castelan@correo.buap.mx
}

Artículo científico recibido: 19 de junio de 2018 aceptado: 12 de noviembre de 2018

RESUMEN. La desertificación es un problema ambiental que amenaza la producción alimentaria, México es uno de los países más afectados por esta problemática. Pocos son los estudios encaminados para conocer los niveles de desertificación actual, y menores los enfocados a prevenir este proceso, dada la susceptibilidad de los territorios para desarrollarla o agravarla. Por lo anterior, el objetivo del trabajo fue evaluar la susceptibilidad ambiental a la desertificación de la microcuenca del río Azumiatla, Puebla, México. Se definieron áreas ambientalmente sensibles a la desertificación (AASD), agrupando indicadores en cuatro índices que definen la calidad del suelo, clima, vegetación y manejo. Se calificaron los atributos: textura de suelo, material parental, fragmentos de rocas en superficie, pendiente, profundidad del suelo, precipitaciones, riesgo de incendio, protección de la flora contra la erosión, resistencia de la vegetación a la sequía, cobertura, intensidad y uso del suelo, así como la aplicación de políticas para su protección. Se utilizó un sistema de información geográfica para generar el mapa final de AASD, así como los mapas de los índices calculados. Los resultados muestran que el $84.6 \%$ del área presenta niveles críticos de sensibilidad a la desertificación, mientras que el $15.4 \%$ presenta fragilidad a la desertificación; siendo las pendientes inclinadas con suelos someros, la siembra de cultivos intensivos anuales y la deforestación, las causas que promueven la degradación del suelo en la microcuenca.

Palabras clave: Aridez, degradación, indicadores, sequía, suelo.

ABSTRACT. Desertification is an environmental problem that threatens food production, with Mexico being one of the countries most affected by this problem. There are few studies aimed at determining current desertification levels, and even fewer focused on preventing this process; however, there is a significant need for such studies given the susceptibility of territories to develop this problem or have it worsen. Therefore, the objective of this study was to evaluate the environmental susceptibility to desertification of the Azumiatla River Micro-watershed, Puebla, Mexico. Areas that are environmentally sensitive to desertification (AESD) were defined, grouping indicators into four indices that define soil quality, climate, vegetation and management. Attributes graded were: soil texture, parent material, surface rock fragments, slope, soil depth, rainfall, fire risk, flora protection against erosion, vegetation resistance to drought, cover, intensity and land use, as well as the application of policies for their protection. A geographic information system was used to generate the final AESD map, as well as the maps of the calculated indices. Results show that $84.6 \%$ of the area presents critical desertification sensitivity levels, while $15.4 \%$ presents fragility to desertification, with steep slopes with shallow soils, sowing of intensive annual crops and deforestation being the main causes that promote soil degradation in the micro-watershed.

Key words: Aridity, degradation, indicators, drought, soil. 


\section{INTRODUCCIÓN}

La desertificación es un tipo de degradación del suelo propia de regiones secas. Este fenómeno es el resultado de interrelaciones entre factores de origen climático y antropogénico (Granados-Sánchez et al. 2013). Los principales agentes biofísicos que provocan la desertificación son la pérdida de cubierta vegetal, la reducción de materia orgánica del suelo, los cambios adversos en las propiedades físicas y químicas del suelo, y la erosión eólica e hídrica (Chen et al. 2002). Socialmente esto se ve reflejado en la inestabilidad económica de los países, resultado de la disminución en la productividad del campo, el aumento en los procesos migratorios de las áreas rurales a las urbanas, la variabilidad en la tasa de pobreza, la marginación de zonas rurales, entre otros (Garay-Romero y Cabero-Diéguez 2013). Por lo que la desertificación es una problemática ambiental y socioeconómica importante en el mundo, que afecta de forma directa el bienestar y futuro de la humanidad (García-Orozco et al. 2012, Granados-Sánchez et al. 2013).

El estado de Puebla por su ubicación geográfica, condiciones climáticas y orográficas, cuenta con amplias extensiones de zonas áridas y semiáridas (SEMARNAT 2012). La microcuenca del río Azumiatla se localiza en la puerta de la mixteca poblana, en la localidad de San Andrés Azumiatla, tiene una precipitación promedio anual de $700 \mathrm{~mm}$ (SSAOT 2011); en la que se pronostica que en medio siglo la precipitación disminuirá un $15 \%$ y la temperatura aumentará $2{ }^{\circ} \mathrm{C}$; lo que ocasionará la disminución en la disponibilidad del agua en un $23 \%$ y aumentará el déficit hídrico en un 55\% (UNCCD 2011). Lo que ocasionará que la actividad agrícola sea afectada por la escasez de agua, lo que se sumará a la pérdida paulatina de fertilidad del suelo (Gómez et al. 2007). Al respecto no se tienen estudios que busquen determinar los niveles de desertificación en la zona de estudio, a pesar de la evidente degradación de los suelos (CONAFOR 2013). A nivel nacional no existe una metodología definida para evaluar procesos de desertificación (GranadosSánchez et al. 2013). Las existentes se basan en el análisis de procesos pedogenéticos y climáticos (CONAFOR 2014), sin importar la influencia que tienen los componentes socio-ambientales sobre este proceso de degradación. Por lo anterior el ojetivo fue evaluar la susceptibilidad ambiental a la desertificación de la microcuenca del río Azumiatla, Puebla, México; aplicando el modelo desarrollado en el proyecto europeo MEDALUS (Mediterranean Desertification and Land Use).

\section{MATERIALES Y MÉTODOS}

La zona de estudio se localiza al sur de la Presa Miguel Ávila Camacho, a $13 \mathrm{~km}$ de la ciudad de Puebla de Zaragoza, México; en las coordenadas geográficas $18^{\circ} 48^{\prime}$ y $18^{\circ} 56^{\prime}$ LN y $98^{\circ} 11^{\prime}$ y $98^{\circ} 17^{\prime}$ LO. Abarca un área aproximada de 5158 ha, tiene sus límites establecidos por el parteaguas de la microcuenca del río Azumiatla (Figura 1). Los tipos de suelo que presenta son Feozem haplico, Rendzina, Vertisol pelico y Litosol; con clima templado subhúmedo con lluvias en verano y precipitación promedio anual de $700 \mathrm{~mm}$. Tiene amplias extensiones de pastizal inducido, agricultura de temporal y relictos de bosque de encino asociado con vegetación arbustiva secundaria (INEGI 2000, SEMARNAT 2012).

Con el apoyo de imágenes satelitales analizadas en un sistema de información geográfica y por recorridos de campo se definieron en la microcuenca tres áreas homogéneas considerando los gradientes de la pendiente de $0-25 \%, 25-50 \%$ y mayor de $50 \%$, y el tipo de relieve. Se establecieron 30 puntos de muestreo, que se caracterizaron con la metodología establecida por Ruiz et al. (1999). También se describieron 15 perfiles de los principales suelos, de los cuales se tomaron muestras de aproximadamente un kilogramo a la profundidad de $30 \mathrm{~cm}$, para luego trasladar las muestras al laboratorio de análisis de suelos agrícolas del Departamento de investigación en Ciencias Agrícolas de la Benemérita Universidad Autónoma de Puebla, para secarlas y tamizarlas para su posterior análisis por triplicado de acuerdo con la Norma Oficial Mexicana NOM021-RECNAT-2000. Con la información generada en campo y laboratorio se aplicó la metodología de 


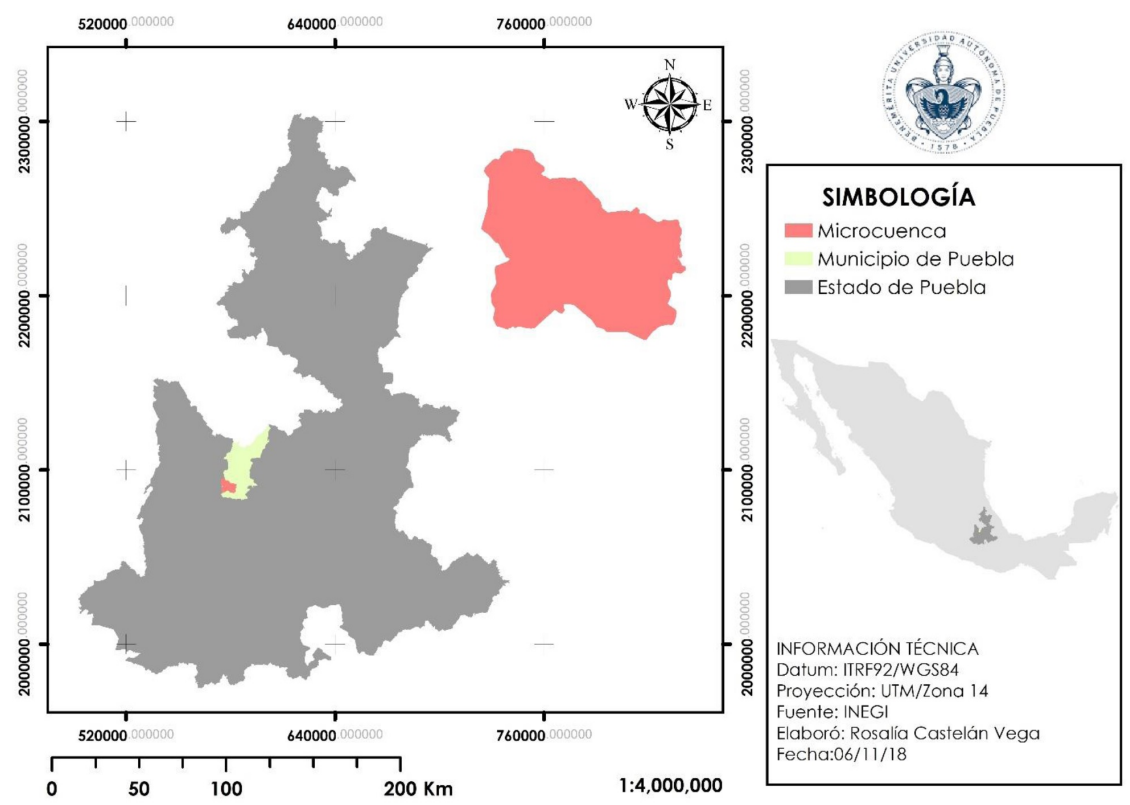

Figura 1. Ubicación de la zona de estudio. Puebla, México.

AASD de Kosmas et al. (1999), que propone evaluar la sensibilidad ambiental del terreno en función de la calidad del suelo, clima, vegetación y manejo, para definir las inidades AASD.

Para calcular el índice de calidad del suelo (ICS) se consideraron los atributos de textura, material parental, fragmentos de roca en superficie, profundidad y gradiente de la pendiente (Tabla 1): ICS $=($ Textura * material parental * fragmento de roca * profundidad * pendiente $)^{1 / 5}$

La calidad del clima se obtuvo del análisis de la información histórica de 30 de las normales promedio de temperatura y precipitación de la estación climatológica de Azumiatla. Para luego calcular el Índice de aridez propuesto por Kosmas et al. (2002), que agrupa en seis clases, con la siguiente ecuación:

$$
I A=\sum_{i=1}^{n}(2 t i-P i) x k
$$

Donde: $\mathrm{IA}=$ índice de aridez, $\mathrm{ti}=$ temperatura media mensual $\left({ }^{\circ} \mathrm{C}\right), \mathrm{Pi}=$ precipitación media mensual (mm), y k = proporción de meses que 2ti $-\mathrm{Pi}>0$.
Tabla 1. Valoración de los factores de calidad del suelo

\begin{tabular}{|c|c|c|}
\hline Descripción & Clase textural & Índice \\
\hline Buena & $A, F, F A, A L, A a$ & 1 \\
\hline Moderada & $\mathrm{L}, \mathrm{FL}, \mathrm{FAL}, \mathrm{Faa}$ & 1.2 \\
\hline Pobre & $\mathrm{aF}, \mathrm{Fa}$ & 1.6 \\
\hline Muy pobre & A & 2 \\
\hline Descripción & Pendiente (\%) & Índice \\
\hline Ondulado & $<25$ & 1 \\
\hline Fuerte & $25-50$ & 1.5 \\
\hline Muy fuerte & $>50$ & 2 \\
\hline Descripción & Tipo de roca & Índice \\
\hline Bueno & $\begin{array}{l}\text { Esquistos, pizarra, básica, ultrabásica, } \\
\text { conglomerados, no consolidados }\end{array}$ & 1 \\
\hline Moderado & $\begin{array}{l}\text { Caliza, mármol, granito, rilita, } \\
\text { ingnibrita, gneiss, limonita, arenisca }\end{array}$ & 1.7 \\
\hline Pobre & Piroclásticos & 2 \\
\hline Descripción & Profundidad (cm) & Índice \\
\hline Profundo & $>75$ & 1 \\
\hline Moderado & $30-75$ & 2 \\
\hline Poco & $15-30$ & 3 \\
\hline Muy poco & $<15$ & 4 \\
\hline Descripción & Fragmentos gruesos (\%) & Índice \\
\hline Alta & $>60$ & 1 \\
\hline Moderada & $20-60$ & 1.3 \\
\hline \multirow[t]{5}{*}{ Baja } & $<20$ & 2 \\
\hline & Índice de calidad del suelo & Índice \\
\hline & Alta calidad & $<1.13$ \\
\hline & Moderada calidad & $1.13-1.45$ \\
\hline & Baja calidad & $>1.46$ \\
\hline
\end{tabular}

$\mathrm{A}=$ arcilloso, $\mathrm{F}=$ franco, $\mathrm{FA}=$ franco arcilloso, $\mathrm{AL}=$ franco limoso, $\mathrm{Aa}=$ arcilloso arenoso, $\mathrm{L}=$ limoso, $\mathrm{FL}=$ franco limoso, $\mathrm{FAL}=$ franco arcilloso limoso, $\mathrm{FAa}=$ franco arcilloso arenoso, $\mathrm{aF}=$ arenoso franco, $\mathrm{Fa}=$ franco arenoso. 
En la Tabla 2 se muestran las relaciones de valoración de los atributos utilizados para calcular el índice de calidad del clima (ICC): ICC = (precipitación ${ }^{*}$ aridez $)^{1 / 2}$

Tabla 2. Valoración de los factores de calidad del clima

\begin{tabular}{cc}
\hline Precipitación $(\mathrm{mm})$ & Índice \\
\hline$>1000$ & 1 \\
$500-1000$ & 2 \\
$<500$ & 4 \\
\hline Aridez & Índice \\
\hline$<50$ & 1 \\
$50-75$ & 1.1 \\
$75-100$ & 1.2 \\
$100-125$ & 1.4 \\
$125-150$ & 1.8 \\
$>150$ & 2 \\
\hline Índice de calidad de clima & Índice \\
\hline Alta calidad & $<1.15$ \\
Moderada calidad & $1.15-1.81$ \\
Baja calidad & $>1.81$ \\
\hline
\end{tabular}

El índice de calidad de la vegetación se valoró en términos de porcentaje y tipo de cobertura de vegetación (Tabla 3 ) obtenidos de imágenes satelitales analizadas en un sistema de información geográfica: ICV = (Riesgo incendio * protección erosión * resistencia a sequía * cobertura de plantas) $)^{1 / 4}$

La información para la valoración del índice de calidad del manejo (ICM) se obtuvo mediante análisis de uso de suelo en sistemas de información geográfica y la realización de entrevistas a campesinos y autoridades de la junta auxiliar de San Andrés Azumiatla sobre los sistemas de manejo agropecuario en la microcuenca, además de la revisión de programas de manejo sustentable por instituciones como la Secretaría del medio ambiente y recursos naturales, Secretaria de desarrollo social, Secretaria de agricultura, ganadería, desarrollo rural y pesca: $\mathrm{ICM}=($ Intensidad de uso * aplicación de política ambiental) $)^{1 / 2}$

Se consideró el establecimiento de cultivos, pastos, zonas naturales y minería o áreas recreativas (Tabla 4). Posterior a la estimación de la calidad del clima, suelo, vegetación y manejo, se utilizaron los cuatro indicadores derivados para calcular el índice de áreas ambientalmente sensibles a la desertifi- cación (IAASD), según la siguiente relación: IAASD $=(\text { ICS * ICC * ICV * ICM })^{1 / 4}$

Los resultados del IAASD muestran los tipos: crítico, frágil, potencial y no afectado. Cada uno se definió en una escala de tres clases: 1 poco sensible, 2 sensible y 3 altamente sensible (Tabla 5). Se elaboraron los mapas espaciales por cada índice generado así como el mapa de las AASD en un sistema de información geográfica (ArcMap 10.5).

\section{RESULTADOS}

El $53.3 \%$ de los suelos tiene textura francolimosa, con buena capacidad de retención de agua, no anegables, provistos de concentraciones adecuadas de materia orgánica y nutrientes para las plantas, pero son susceptibles de erosión hídrica. El $33.3 \%$ tienen textura franco-arcillosa, desde el punto de vista agronómico en estos suelos las plantas crecen con facilidad por las condiciones de porosidad, aireación y humedad. Mientras que el $13.33 \%$ presenta textura arcillosa, lo que indica que son suelos que se anegan con mucha facilidad en la época de lluvias, son poco porosos y desde el punto de vista agronómico, son difíciles de labrar (Tabla 6).

El $66.1 \%$ de los suelos de la microcuenca se han desarrollado de rocas ígneas extrusivas, el $18.8 \%$ de caliza, el $14.4 \%$ de areniscas y conglomerados, y el $0.7 \%$ de material aluvial. En lo que respecta a la inclinación de la pendiente, aproximadamente el $30 \%$ de la superficie presente relieve alomado con 30 a $40 \%$ de inclinación, el $45 \%$ tiene pendientes superiores al $20 \%$, y el $25 \%$ de la superficie tiene pendientes mayores al $10 \%$; por lo que bajo condiciones de deforestación el relieve constituye un factor de riesgo de erosión en la microcuenca. Los suelos son moderadamente profundos en las zonas con pendiente de $25 \%$ y con pastizal $(30 \mathrm{a} 75 \mathrm{~cm})$ y profundos $(>75 \mathrm{~cm}$ ) en el valle y las áreas cubiertas con vegetación.

Con respecto al clima (Figura 2), la precipitación promedio de un año es de $577.10 \mathrm{~mm}$ de abril a octubre, ocurriendo el $89.20 \%$ de las precipitaciones en un período de 4 meses con alta intensidad, por lo que en pendientes pronunciadas y bajo condi- 
Castelán-Vega et al.

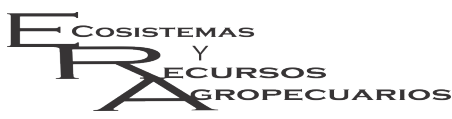

Desertificación en San Andrés Azumiatla

Ecosist. Recur. Agropec. 6(16):91-101,2019

Tabla 3. Valoración de los factores de calidad de la vegetación.

\begin{tabular}{lcc}
\hline Descripción & Riesgos de incendio & Índice \\
\hline Baja & Suelo desnudo, cultivos agrícolas perennes y anuales (maíz, tabaco) & 1 \\
Moderada & Cultivos agrícolas anuales (cereales, pastos), bosques (deciduos, mixto, siempre verde) & 1.3 \\
Alta & Pastos permanentes & 1.6 \\
Muy alta & Bosques de pinos & 2 \\
\hline Descripción & Protección contra la erosión & Índice \\
\hline Muy alta & Bosque siempre verde, bosque mixto & 1 \\
Alta & Pastos permanentes, cultivos agrícolas, perennes siempre verde & 1.3 \\
Baja & Cultivos agrícolas perennes deciduos y semideciduos & 1.8 \\
Muy baja & Cultivos agrícolas anuales (cereales) y pastos anuales & 2 \\
\hline Descripción & Resistencia a la sequía & Indice \\
\hline Muy alta & Bosque siempre verde, bosque mixto & 1 \\
Alta & Bosque deciduo, bosque de galería & 1.2 \\
Moderada & Cultivos agrícolas perennes & 1.4 \\
Baja & Pastos perennes & 1.7 \\
Muy baja & Cobertura (\%) & 2 \\
\hline Descripción & $>40$ & Índice \\
\hline Alta & 10 - 40 & 1 \\
Baja & $<10$ & 1.8 \\
Muy baja & Cultivos agrícolas anuales (cereales, hortalizas) y pastos anuales & 2 \\
\hline & Indice de calidad de la vegetación & Indice \\
\hline & Alta calidad & $<1.13$ \\
& Moderada calidad & $1.13-1.38$ \\
& Baja Calidad & $>1.38$ \\
\hline
\end{tabular}

Tabla 4. Valores de los factores de calidad del manejo.

\begin{tabular}{lcc}
\hline Descripción & Intensidad de uso & Índice \\
\hline 1 & Baja & 1 \\
2 & Mediana & 1.5 \\
3 & Alta & 2 \\
\hline Descripción & Políticas & Índice \\
\hline Completo & $>75 \%$ del área bajo protección & 1 \\
Parcial & $25-75 \%$ del área bajo protección & 1.5 \\
Incompleto & $<25 \%$ del área bajo protección & 2 \\
\hline & Índice de calidad del manejo & Índice \\
\hline & Alta calidad & $1.0-1.25$ \\
& Moderada calidad & $1.26-1.50$ \\
& Baja calidad & $>1.51$ \\
\hline
\end{tabular}

Tabla 5. Índice de áreas ambientalmente sensibles a la desertificación (IAASD).

\begin{tabular}{lcc}
\hline Tipo & Subtipo & IAASD \\
\hline Crítico & C3 $(\mathrm{as})$ & $>1.53$ \\
Crítico & $\mathrm{C} 2(\mathrm{~s})$ & $1.42-1.52$ \\
Crítico & $\mathrm{C} 1(\mathrm{ps})$ & $1.38-1.41$ \\
Frágil & $\mathrm{F} 3(\mathrm{as})$ & $1.33-1.37$ \\
Frágil & $\mathrm{F} 2(\mathrm{~s})$ & $1.27-1.32$ \\
Frágil & $\mathrm{F} 1(\mathrm{ps})$ & $1.23-1.26$ \\
Potencial & $\mathrm{P}$ & $1.17-1.22$ \\
No afectado & $\mathrm{N}$ & $<1.17$ \\
\hline \multicolumn{2}{l}{ as= altamente sensible; $\mathrm{s}=$ sensible; $\mathrm{pS}=$} \\
poco sensible.
\end{tabular}

ciones de deforestación, se favorece la escorrentía superficial, lo que ocasiona la erosión de los sue- los. La época de sequía inicia en noviembre y termina en marzo, con déficit hídrico de octubre a mayo. Debido a que la evapotranspiración es superior a la precipitación, se detiene el crecimiento y desarrollo de la vegetación, a lo que se suma la clase textural que predomina en la microcuenca, con lo que restringe más la disponibilidad de humedad del suelo.

Con respecto al uso de suelo y vegetación, el $48.3 \%$ de la superficie total de la microcuenca se dedica a la agricultura de temporal con cultivo de maíz, avena y calabaza, actividad favorecida por apoyos gubernamentales aportados por el programa Alianza para el Campo. El $37.5 \%$ de la superficie corresponde a pastizal, que se utiliza para la actividad pecuaria y por el abandono del campo, ya que algunos agricultores prefirieron migrar a las áreas urbanas circundantes $u$ otros países, para incrementar su ingreso económico. De la superficie total de la microcuenca el $14.2 \%$ corresponde a pequeños relictos de bosque de encino, que se localizan en las partes más altas de la microcuenca.

\section{DISCUSIÓN}

La presencia de pendientes altamente incli- 
Tabla 6. Clase textural de los perfiles analizados.

\begin{tabular}{lccccl}
\multirow{2}{*}{ Perfil } & $\begin{array}{c}\text { Profundidad } \\
(\mathrm{cm})\end{array}$ & \multicolumn{3}{c}{ Textura (\%) } & \multirow{2}{*}{ Clase textural } \\
\cline { 3 - 5 } & $0-30$ & Arena & Arcilla & Limo & \\
\hline 1 & $0-38.4$ & 5.3 & 56.3 & Franco-limosa \\
2 & 32.2 & 3.2 & 64.6 & Franco-limosa \\
3 & $0-30$ & 34.1 & 3.1 & 62.8 & Franco-limosa \\
4 & $0-30$ & 38.7 & 1.2 & 60.1 & Franco-limosa \\
5 & $0-30$ & 38.4 & 0.9 & 60.7 & Franco-limosa \\
6 & $0-30$ & 30.6 & 0.63 & 68.77 & Franco-limosa \\
7 & $0-30$ & 32.4 & 1.18 & 66.42 & Franco-limosa \\
8 & $0-30$ & 34.7 & 5.16 & 60.14 & Franco-limosa \\
9 & $0-30$ & 32 & 39.8 & 28.2 & Franco-arcillosa \\
10 & $0-30$ & 32 & 39.8 & 28.2 & Franco-arcillosa \\
11 & $0-30$ & 34.2 & 28.52 & 37.28 & Franco-arcillosa \\
12 & $0-30$ & 50.92 & 29.8 & 19.28 & Franco-arcillosa \\
13 & $0-30$ & 37.84 & 38.88 & 23.28 & Franco-arcillosa \\
14 & $0-30$ & 24.92 & 59.8 & 15.28 & Arcillosa \\
15 & $0-30$ & 37.84 & 43.24 & 18.92 & Arcillosa \\
\hline
\end{tabular}

\section{Climograma estación Azumiatla, Puebla}

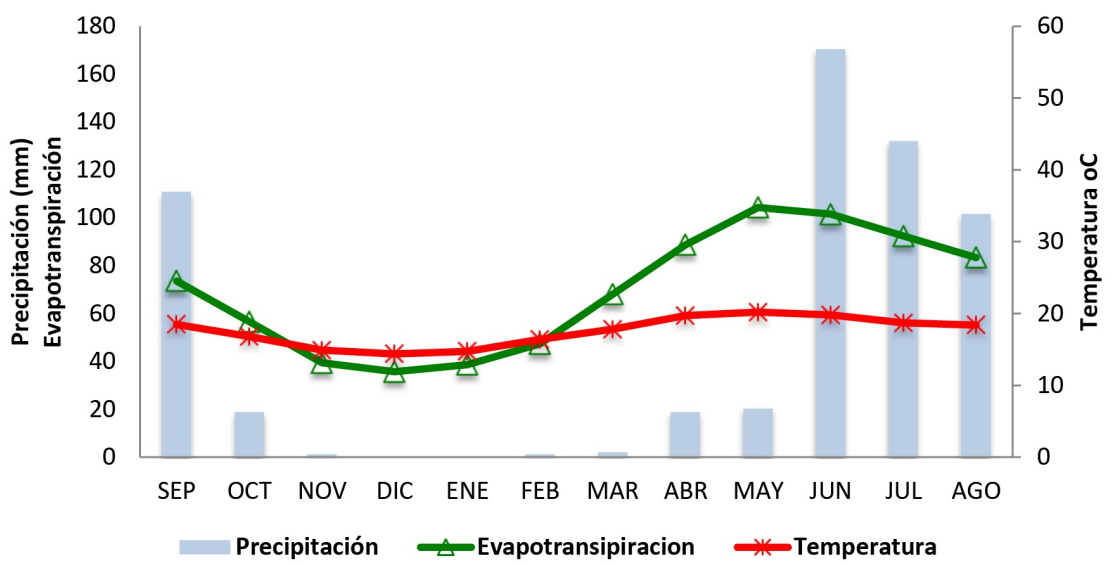

Figura 2. Climograma de la estación meteorológica Azumiatla.

nadas y la deforestación de la zona de estudio, favorecen los procesos de erosión acelerados, lo que ocasiona baja calidad del suelo en el $68 \%$ de microcuenca. Por otro lado, las condiciones de textura del suelo y las reducidas áreas con poca inclinación de la pendiente, son los factores determinantes que favorecen que el $32 \%$ de los suelos de la microcuenca presenten calidad moderada, no registrándose suelos de alta calidad (Tabla 7, Figura 3). La microcuenca se categoriza, en base al índice ombrotérmico de Bagnouls-Gaussen (Llanas y Contreras 2009) como una zona seca (112), por lo que la calidad del clima bajo este modelo de evaluación es moderada en toda la microcuenca. El uso de suelo que predomina en la microcuenca es agrícola (48.3\%), en donde las condiciones de manejo no son la adecuadas bajo pendientes con alta inclinación, con siembras sin medidas de conservación de suelo, y con labranza en el mismo sentido de la pendiente, por lo que el arrastre del suelo es facilitado por la gravedad, y se acelera durante los periodos de lluvia intensa, lo que incrementa los procesos erosivos de la región (Mehuys et al. 2009, Bakr et al. 2012).

Las zonas con pastizales (37.5\%) brindan cobertura en el periodo de lluvias, pero en contraparte los pastizales tienen raíces poco profundas, que bajo precipitaciones torrenciales $y$ laderas pronunciadas, no logran detener el agua de 
Tabla 7. Índice de calidad de los suelo de la microcuenca.

\begin{tabular}{cccccc}
\hline Perfil & $\begin{array}{c}\text { Profundidad } \\
\text { Perfil }(\mathrm{cm})\end{array}$ & Material parental & $\begin{array}{c}\text { Fragmentos } \\
\text { de roca }(\%)\end{array}$ & $\begin{array}{c}\text { Pendiente } \\
(\%)\end{array}$ & ICS \\
\hline 1 & 70 & Ígnea extrusiva & 30 & 30 & 1.48 \\
2 & 75 & Ígnea extrusiva & 2 & 25 & 1.61 \\
3 & 70 & Ígnea extrusiva & 25 & 30 & 1.61 \\
4 & 75 & Ígnea extrusiva & 20 & 40 & 1.61 \\
5 & 90 & Ígnea extrusiva & 25 & 20 & 1.48 \\
6 & 80 & Ígnea extrusiva & 25 & 25 & 1.48 \\
7 & 85 & Ígnea extrusiva & 30 & 30 & 1.48 \\
8 & 85 & Ígnea extrusiva & 20 & 20 & 1.48 \\
9 & 90 & Ígnea extrusiva & 20 & 15 & 1.43 \\
10 & 40 & Arenisca-conglomerados & 35 & 15 & 1.47 \\
11 & 8 & Caliza & 20 & 12 & 1.83 \\
12 & 10 & Caliza & 25 & 12 & 1.83 \\
13 & 8 & Caliza & 15 & 15 & 1.83 \\
14 & 80 & Aluvial & 5 & 2 & 1.52 \\
15 & 60 & Aluvial & 10 & 2 & 1.32 \\
\hline
\end{tabular}

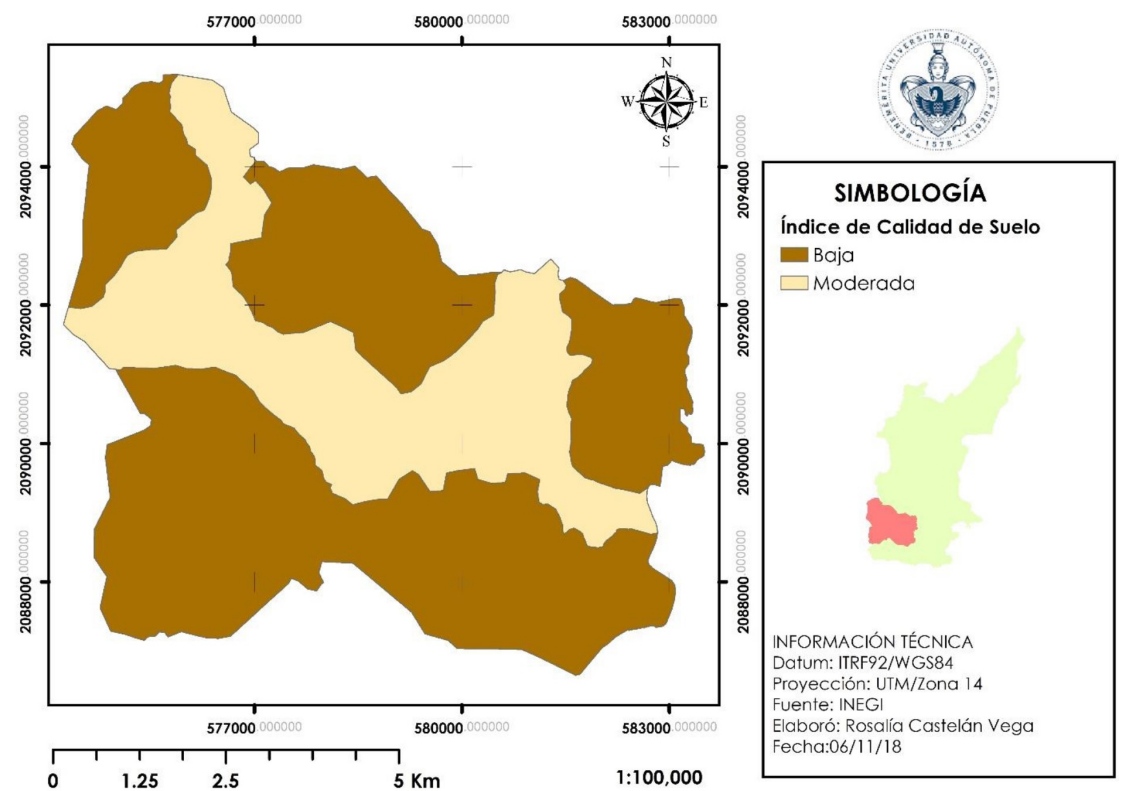

Figura 3. Distribución espacial de la calidad de suelo en la microcuenca.

escorrentía; por lo que a la erosión, se suma el hecho que la vegetación herbácea es altamente susceptible a riesgo de incendio, lo que incrementa la posibilidad de transmisión de fuego hacia los cultivos agrícolas y localidades aledañas, efecto reportado por Durán et al. (2014). El tipo de vegetación que predomina en la microcuenca se cataloga como de alto riesgo a la desertificación (Figura 4), por lo que la calidad es baja en el $85.8 \%$ y alta en el $14.2 \%$. La calidad de manejo es muy baja en el $78 \%$ de la microcuenca, primordialmente en las áreas con pastizal y de mayor inclinación del terreno donde se ubican los cultivos de temporal como el maíz sin práctica de conservación (Figura 5).

El área de bosque de encino presentó baja intervención humana, exhibiendo una moderada calidad de manejo con adecuada protección contra la erosión. El maíz es el principal cultivo sembrado, ya que su producción se utiliza no sólo para la alimentación familiar, sino también para la de bovinos, 


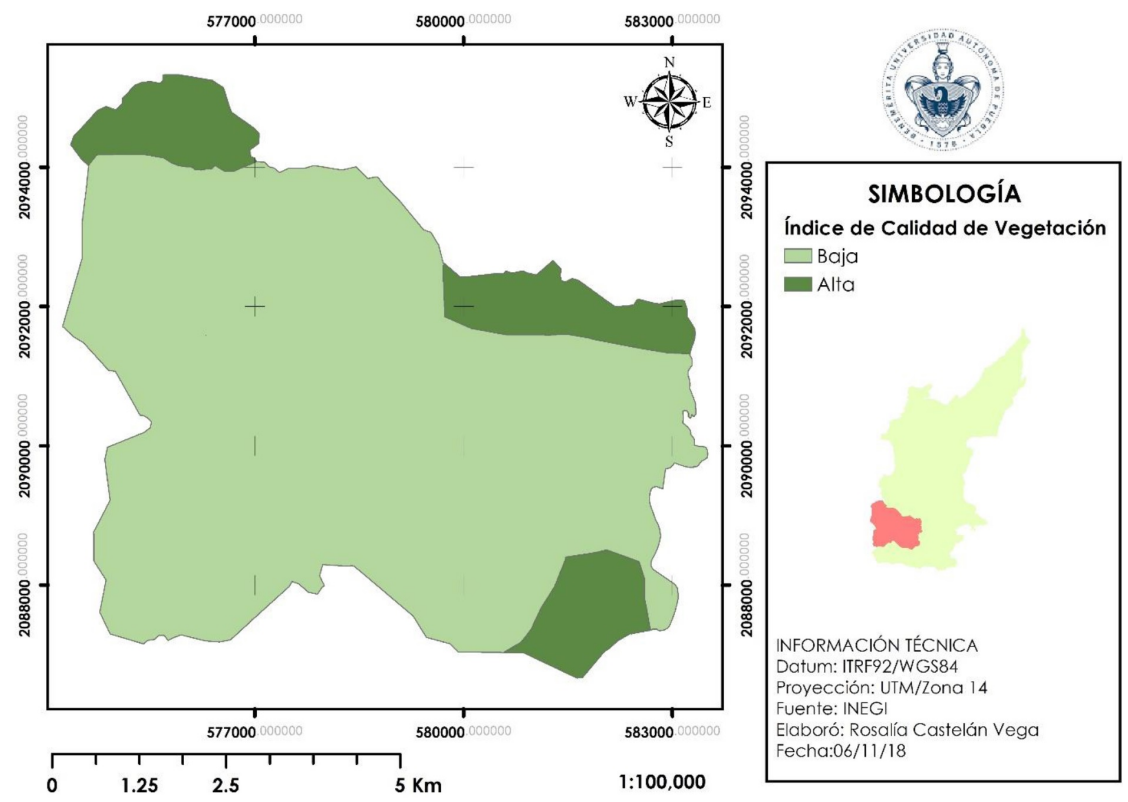

Figura 4. Distribución espacial de la calidad de la vegetación en la microcuenca.

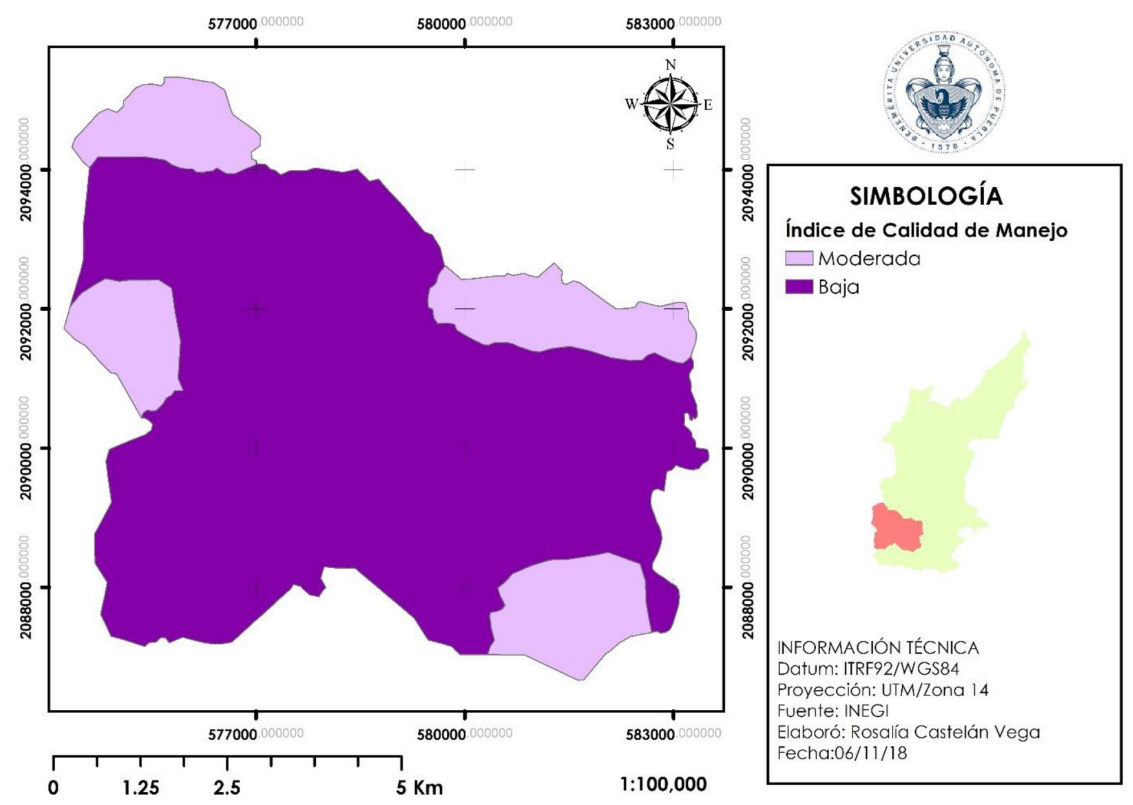

Figura 5. Distribución espacial de la calidad del manejo en la microcuenca.

aves y otros animales domésticos (Hernández-Flores et al. 2009).

En base en la revisión sobre las políticas de conservación aplicadas en la zona de estudio por parte de instituciones como la Secretaria del Medio
Ambiente y Recursos Naturales y la Secretaria de Desarrollo Urbano y Sustentabilidad, se detectó carencia de apoyos en la microcuenca, al igual que la ausencia de programas que promuevan la agricultura sostenible en el municipio, las acciones y estrate- 


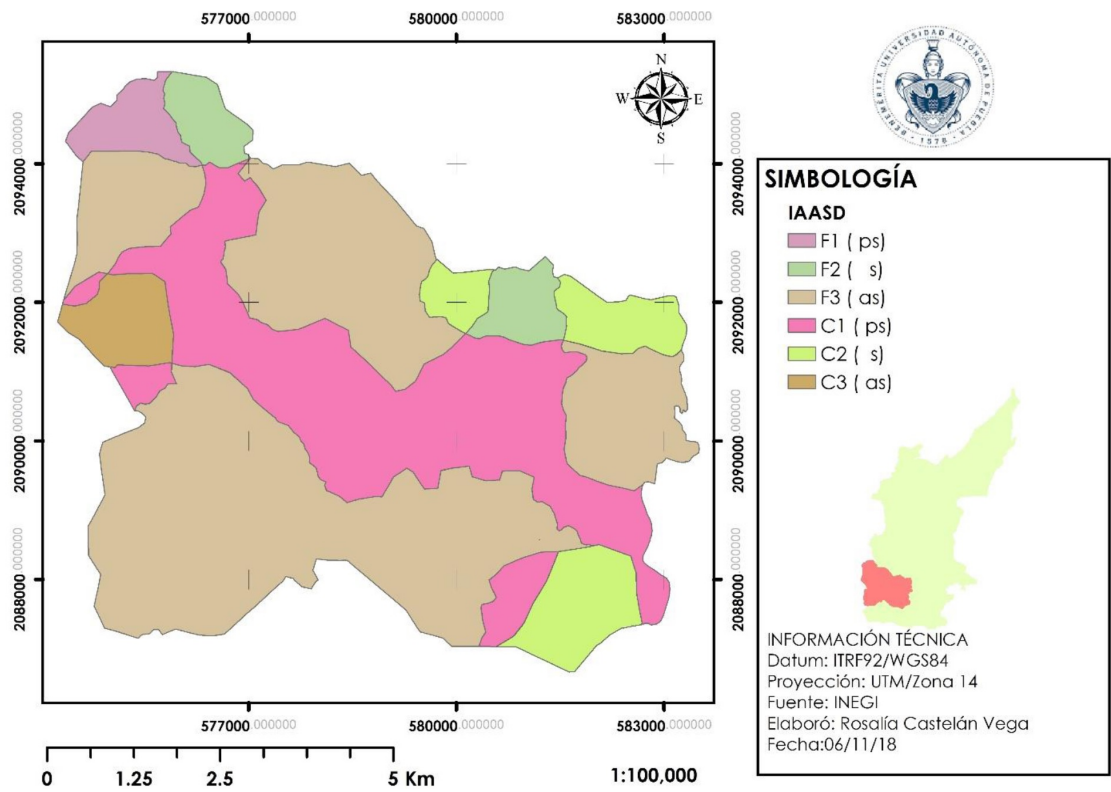

Figura 6. Áreas ambientalmente sensibles a la desertificación de la microcuenca.

gias se han centrado en la expansión de las zonas urbanas e industriales, pero se han olvidado del campesino, lo que coincide con Gómez et al. (2007). Los pocos recursos que llegan son de manera esporádica y desarticulada, aunque hay una incipiente conciencia entre las autoridades municipales de la importancia de los proyectos productivos de carácter sostenibles que incidan en la producción de alimentos y en la creación de fuentes de empleo e ingreso permanentes, con lo que se podría mejorar las condiciones de vida de la población rural (HernándezFlores et al. 2009). La asesoría técnica, capacitación y seguimiento permanente de programas y proyectos de impacto comunitario por parte de las instituciones en la microcuenca son pocos, lo que ha ocasionado que los campesinos no adopten nuevas tecnologías ni avancen en el mejor aprovechamiento de sus recursos naturales (Tapia 2002).

En la Figura 6 se observa la distribución espacial de las áreas ambientalmente sensibles a la desertificación en la microcuenca evaluada, el $84.6 \%$ de la microcuenca tiene categoría crítica y el $15.4 \%$ es frágil, sin zonas sin amenaza de desertificación. Las áreas consideradas como críticas: C1 (3.20\%),
C2 (28.07\%) y C3 (53.38\%), tienen topografía marginal, pastizales, nula vegetación arbórea y escasa profundidad del suelo, así como cultivos intensivos que promueven la degradación del suelo, resultados similares fueron reportados por Saleh et al. (2018) con el mismo modelo de evaluación. Las zonas frágiles (F1 (2.59\%), F2 (4.44\%) y F3 (8.33\%) son sensibles a la degradación sólo cuando se presenten cambios drásticos de uso del suelo, en el que se promueva la disminución del potencial biológico, con la consecuente pérdida de cobertura vegetal y elevadas tasas de erosión (Vieira et al. 2015). Estas zonas actualmente se encuentran amenazadas por deforestación para la introducción de cultivos anuales, por lo que es común la quema de pastos en la frontera agrícola, lo que aumenta los riesgos de incendio (Jafari y Bakhshandehmehr 2015). Por lo que necesario conservar las zonas con relictos de bosque, así como restringir la actividad agrícola para evitar su expansión.

\section{CONCLUSIONES}

El $84.6 \%$ de la microcuenca, presenta sensi- 
bilidad ambiental a la desertificación, mientras que el $15.4 \%$ tiene fragilidad, debido a la deforestación y la agricultura en pendientes sin medidas de conservación. El $68 \%$ de los suelos son someros y con erosión, por lo que son de baja calidad. El clima en toda la microcuenca es de calidad moderada, ya que la escasa precipitación que presenta junto con su alto índice de aridez la condicionan a ser más susceptible que las cuencas templadas al desarrollo de procesos de degradación que conllevan a la desertificación. El $85.8 \%$ de la vegetación es baja y alta en el $14.2 \%$, debido a los relictos de bosque de encino que permanecen, se tiene baja calidad de manejo, por la falta de programas que capaciten en prácticas agrícolas sostenibles y la promoción de la restauración ecológica. Se requiere implementar prácticas de conservación que mitiguen y reduzcan el impacto de la actividad agrícola.

\section{LITERATURA CITADA}

Bakr N, Weindorf D, Bahnassy M, El-Badawi M (2012) Multi-temporal assessment of land sensivity to desertification in a fragile agro-ecosystem: Environmental indicators. Ecological indicators 15: 271-280.

Chen J, Chen JZ, Tan MZ, Gong ZT (2002) Soil degradation: a global problem endangering sustainable development. Journal of Geographical Sciences 12: 243-252.

CONAFOR (2013) Línea base nacional de degradación de tierras y desertificación. CONAFOR. México. 152p.

CONAFOR (2014) Protocolo para el sistema nacional de monitoreo de tierras con degradación y desertificación. CONAFOR. México. 130p.

Durán Z, Rodríguez R, Cuadros S, Francia R (2014) Impacto de la erosión y escorrentía en laderas de agroecosistemas de montaña mediterráneos. Ecosistemas 23: 66-72.

Garay-Romero IC, Cabero-Diéguez V (2013) El aspecto social de la desertificación: Análisis de la percepción de la administración pública en Centro América. Gestión y Ambiente 16: 37-48.

García-Orozco JA, Cedillo-Gutiérrez JC, Juan-Pérez JI, Balderas-Plata MA (2012) Procesos de cambio en el uso del suelo de una microcuenca en el altiplano mexicano. El caso del Río San José en el Estado de México. Papeles de Geografía 55: 63-73.

Granados-Sánchez D, Hernández-García MA, Vázquez-Alarcón A, Ruíz-Puga P (2013) Los procesos de desertificación y las regiones áridas. Revista Chapingo Serie Ciencias Forestales y del Ambiente 19: 45-66.

Gómez C, Martínez C, Marroni G (2007) Relaciones de género en procesos migratorios periurbanos en Puebla. Ra Ximhai 3: 621-648.

Hernández-Flores JA, Martínez-Corona B, Méndez-Espinoza JA, Pérez-Avilés R, Ramírez-Juárez J, NavarroGarza H (2009) Rurales y periurbanos: una aproximación al proceso de conformación de la periferia poblana. Papeles de Población 15: 275-295.

INEGI (2000) Síntesis geográfica del Estado de Puebla. Anexo cartográfico. Edit. INEGI. México. 225p.

Jafari R, Bakhshandehmehr (2015) Quantitative mapping and assessment of environmentally sensitive áreas to desertification in central Iran. Land Degradation \& Development 27: 108-119.

Kosmas C, Ferrara A, Briassouli H, Imeson I (1999) Methodology for mapping ESAs to desertification. In: Kosmas C, Kirkby M, Geeson M (ed.) The medalus project Mediterranean desertification and land use. Manual on key indicators of desertification and mapping environmentally Sensitive Areas to desertification. Brussels, Belgium. pp: 31-47. 
Kosmas C, Gerontidis S, Tsara M, Marantianou M (2002) Defining environmentally sensitive areas to desertification: An application to the Island of Lesvos. Proceedings of the Third International Congress Man and Soil at the Third Millennium. Geoforma 2: 1233-1253.

Llanas RJ, Contreras CS (2009) Variabilidad climatológica en la ciudad de San Luis Potosí durante el período 1878-2000 y su relación con el fenómeno de El Niño. Boletín de los Sistemas Nacionales Estadísticos y de Información Geográfica 2: 249-272.

Mehuys G, Tiessen K, Villatoro M, Sancho F, Lobb D (2009) Erosión por labranza con arado de disco en suelos volcánicos de ladera en costa rica. Agronomía Costarricense 33: 205-215.

NOM-021-RECNAT-2000. Norma Oficial Mexicana que establece las especificaciones de fertilidad, salinidad y clasificación de suelos. Estudio, Muestreo y Análisis. SEMARNAT, 78 p.

Ruiz J, Calderón E, Tamariz V, Tremols J, Cruz A, Valera MA, et al. (1999) Manual para la descripción de perfiles de suelos y evaluación del entorno. Textos BUAP. Puebla, México. 62p.

Saleh A, Belal A, Jalhoum M (2018) Quantitative assessment of environmental sensitivity to desertification in Sidi Abdel-Rahman Area, Northern west coast of Egypt. Egyptian Journal of Soil Science 58: 13-26.

SEMARNAT (2012) Informe de la Situación del Medio Ambiente en México. SEMARNAT, México. 325p.

SSAOT (2011) Estrategia de mitigación y adaptación del estado de Puebla ante el cambio climático. Secretaría de Sustentabilidad Ambiental y Ordenamiento Territorial. Puebla, México. 308p.

Tapia NA (2002) El proceso de investigación y transferencia de tecnología en el sector agrícola. La experiencia del INIFAP. Aportes 20: 179-183.

UNCCD (2011) Desertication. A visual synthesis. UNCCD-Zoï Environment Network France. Brussels, Belgium. $102 p$.

Vieira R, Tomasella J, Alvalá R, Sestini M, Affonso A, Rodríguez D, et al. (2015) Identifying areas susceptible to desertification in the Brazilian northeast. Solid Earth 6: 347-360. 
